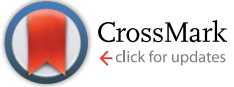

Cite this: RSC Adv., 2015, 5, 94236

Received 9th September 2015 Accepted 23rd October 2015

DOI: $10.1039 / c 5 r a 18450 a$

www.rsc.org/advances

\section{Fluorescent magnesium nanocomplex in a protein scaffold for cell nuclei imaging applications $\dagger$}

\author{
Alok Pandya, ${ }^{a}$ Apritam Tripathi, ${ }^{a}$ Rahul Purohit, ${ }^{a}$ Sanjay Singh, ${ }^{a}$ Manjula I. Nandasiri, \\ Ajay Karakoti, ${ }^{\text {ab }}$ Surinder P. Singh ${ }^{\star c}$ and Rishi Shanker ${ }^{\star a}$
}

Herein, we report a facile strategy for the synthesis of a water-soluble ultra-fine blue-green emitting fluorescent magnesium nanoparticleprotein complex $(\mathrm{MgNC})$. This $\mathrm{MgNC}$ is demonstrated to exhibit excellent photostability and biocompatibility. It was also observed that MgNCs stain cell nuclei with high specificity.

Ultrasensitive fluorescence based imaging techniques have received considerable attention in the visualization of cell organelles or study of intracellular processes in cell biology and molecular spectroscopy. ${ }^{1}$ These techniques rely on bright fluorescent probes attached to DNA, proteins, or other biological components. Particularly, in cellular processes, the specific staining of cellular compartments is highly desirable for imaging subcellular structures to distinguish dissimilar cellular compartments and also to understand localization of the cell organelles. Therefore, researchers continue to utilize the best available options in fluorescence techniques for in vivo imaging of cellular processes. Among many cellular interactions, the imaging of the nucleus is particularly important because it contains deoxyribonucleic acid (DNA), which dictates most intracellular process and also it is the site for DNA replication and drug-DNA interactions. ${ }^{1,2}$ Many sensitive probes such as organic dyes and transition metal complexes have been used for imaging of the cells. ${ }^{3-7}$ However, their cost and limited $\mathrm{pH}$ range restricts their use for imaging in cells.

QDs (such as CdSe, CdTe, and PbTe) have received broad attention due to their unique optical properties and

\footnotetext{
Institute of Life Sciences, School of Science and Technology, Ahmedabad University, Navrangpura, Ahmedabad-380009, India. E-mail: rishi.shanker@ahduni.edu.in; Fax: +91-79-26302419; Tel: +91-79-26302414-18

${ }^{b}$ Institute of Engineering and Technology, School of Science and Technology, Ahmedabad University, Navrangpura, Ahmedabad-380009, India

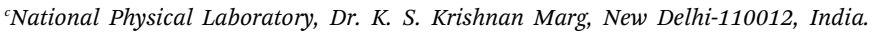
E-mail: singh.uprm@gmail.com

${ }^{d}$ Environmental and Molecular Sciences Laboratory, PNNL, Richland, WA 99354, USA $\dagger$ Electronic supplementary information (ESI) available. See DOI: 10.1039/c5ra18450a
}

biocompatibility. However, the applications of QDs are limited due to the release of $\mathrm{Cd}^{2+}, \mathrm{Pb}^{2+}$, or other heavy metal ions that exert cytotoxicity in in vivo systems and also potential environmental hazard. ${ }^{6,7}$ In past few years, extensive studies have been performed on luminescent $\mathrm{Au}$ (gold) and $\mathrm{Ag}$ (silver) nanoclusters or nanocomplex (NC) using various templates such as peptides, dendrimers, polymers, and proteins. ${ }^{8-13}$ The nanocomplex are attractive because of their high fluorescence, good photostability, less toxicity, good biocompatibility and solubility. ${ }^{13 e}$ Most recently, biomolecules such as protein, enzyme, and peptide capped copper NC and carbon dots have been used in the biomedical imaging owing to their multicolor emission capabilities and non-toxic nature. ${ }^{14}$

It is a well known fact that nucleic acids have affinity to bind divalent ions such as magnesium and stabilise DNA and RNA structure. ${ }^{15}$ Magnesium ions have a critical role in maintaining the positional integrity of closely clustered phosphate groups. These clusters appear in numerous and distinct parts of the cell nucleus and cytoplasm, which coordinates with magnesium ion to form aggregates. ${ }^{16}$ This prompted us to explore development of bioinspired magnesium nanoparticles for imaging of cell nucleus, which is the most important organelle controlling in the growth, proliferation, and apoptosis of a cell. Nevertheless, only a few experimental studies have given direct insight into magnesium nanoparticles ${ }^{17}$ primarily because of the difficulty in preparing highly stable and extremely tiny $\mathrm{Mg}$ nanoparticles. Additionally, subnanometer sized $\mathrm{Mg}$ intrinsically suffers from unstable colloidal dispersion of its particles. So, it is of great interest to develop stable, highly biocompatible and fluorescent magnesium nanoparticles complexed with protein scaffold with visible emission. This MgNC was also observed to stain cell nuclei with high specificity.

Exclusively, we report for the first time synthesis of biocompatible, ultrafine MgNC by a simple one-pot chemical reduction method using bovine serum albumin (BSA) as protein matrix (Fig. 1). It has been demonstrated that BSA can be used as the model protein for the synthesis and stabilization of metal nanostructures. ${ }^{14 b}$ The as-synthesized bioconjugated MgNC 
exhibit emission and has been characterized using various spectroscopic and microscopic techniques. Further, using fluorescence microscopy, we studied the cellular uptake of MgNC, including uptake kinetics and intracellular localization.

A typical UV-Vis absorption spectrum of an aqueous solution containing free BSA and $\mathrm{MgNC}$ is presented in Fig. 2A. The absorption peak at $280 \mathrm{~nm}$ for bare BSA is attributed to the $\pi-\pi^{*}$ transition of aromatic amino acid residue characteristic of polypeptide backbone structure $\mathrm{C}=\mathrm{O}$, whereas the absorption peaks at 292 and $322 \mathrm{~nm}$ indicate the shift in $\pi-\pi^{*}$ transition and is attributed to the formation of MgNC (Fig. 2A). The formation of any kind of oxidation product of the amino acids

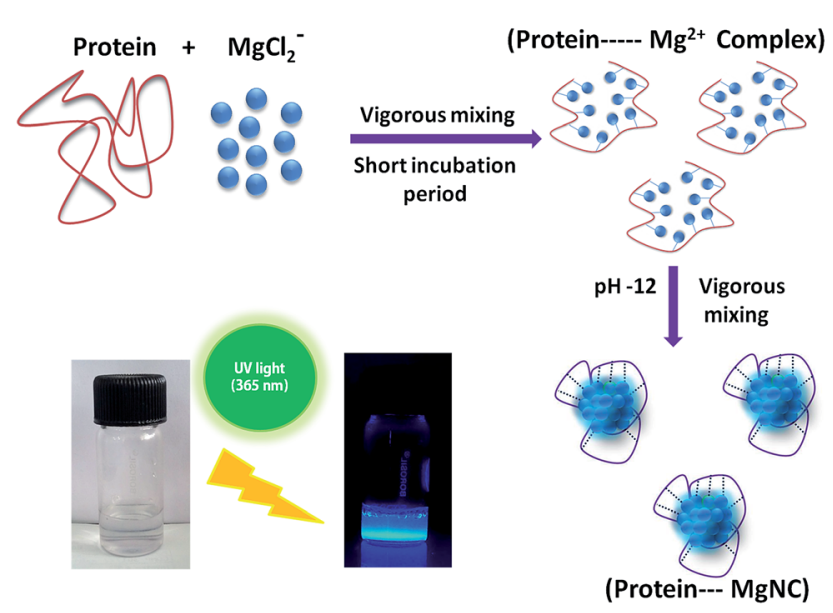

Fig. 1 The facile synthetic route for producing BSA mediated synthesis of $\mathrm{MgNC}$ (inset: capped MgNC emitting blue-green fluorescence when excited with a handheld UV lamp (365 nm)). in protein is ruled out as also discussed in studies from other research groups. ${ }^{14 b}$ The observed $322 \mathrm{~nm}$ absorption peak as well as the emission maxima revealed in the present study is purely due to the formation of MgNC. The low and high resolution transmission electron microscopy (HRTEM) image (Fig. 2E and F) shows average size of these ultrafine monodispersed MgNC of size $\sim 8.5 \mathrm{~nm}$ (inset $2 \mathrm{E}$ ) which was calculated using the software (Image-J). Crystal lattice fringes with 2.8 A spacing indicates the (100) planes of the metallic $\mathrm{Mg}$ (inset 2F). Further the average hydrodynamic size of MgNCs analyzed by DLS measurements was found to be $12 \pm 0.7 \mathrm{~nm}$. The observed change in size compared to TEM is attribute to protein swelling and fragmentation to accommodate MgNC. Protein fragmentation with multimodal distribution for the coexistence of MgNC and free protein as well as enhancement of aggregation is also observed (Fig. S1†). It is well known that fragmentation of protein can occur when the $\mathrm{pH}$ of the environment changes drastically. ${ }^{14 b}$ All together, these confirms the formation of protein-magnesium nanocomplex.

Powder X-ray diffraction (PXRD) of MgNC shows the crystalline nature of the MgNC and matched with the face-centered cubic structure of metallic magnesium (Fig. 2B). The PXRD spectrum contains multiple peaks which are clearly distinguishable. X-ray diffraction patterns of as-synthesized MgNC match with diffraction patterns of hexagonal $\mathrm{Mg}$ (solid black line, JCPDS 04-0770). Peaks located at $2 \theta=23.3^{\circ}, 31.6^{\circ}, 33.6^{\circ}$, $37.8^{\circ}, 45.3^{\circ}, 56.4^{\circ}, 66.1^{\circ}$ and $75.2^{\circ}$ are assigned to the (001), (100), (002), (101), (102), (110), (202) and (104) planes of hexagonal magnesium, respectively. No significant diffraction peak is observed for magnesium oxide phase (JCPDS 89-7746) or any other magnesium phase except hexagonal $\mathrm{Mg}$ indicating
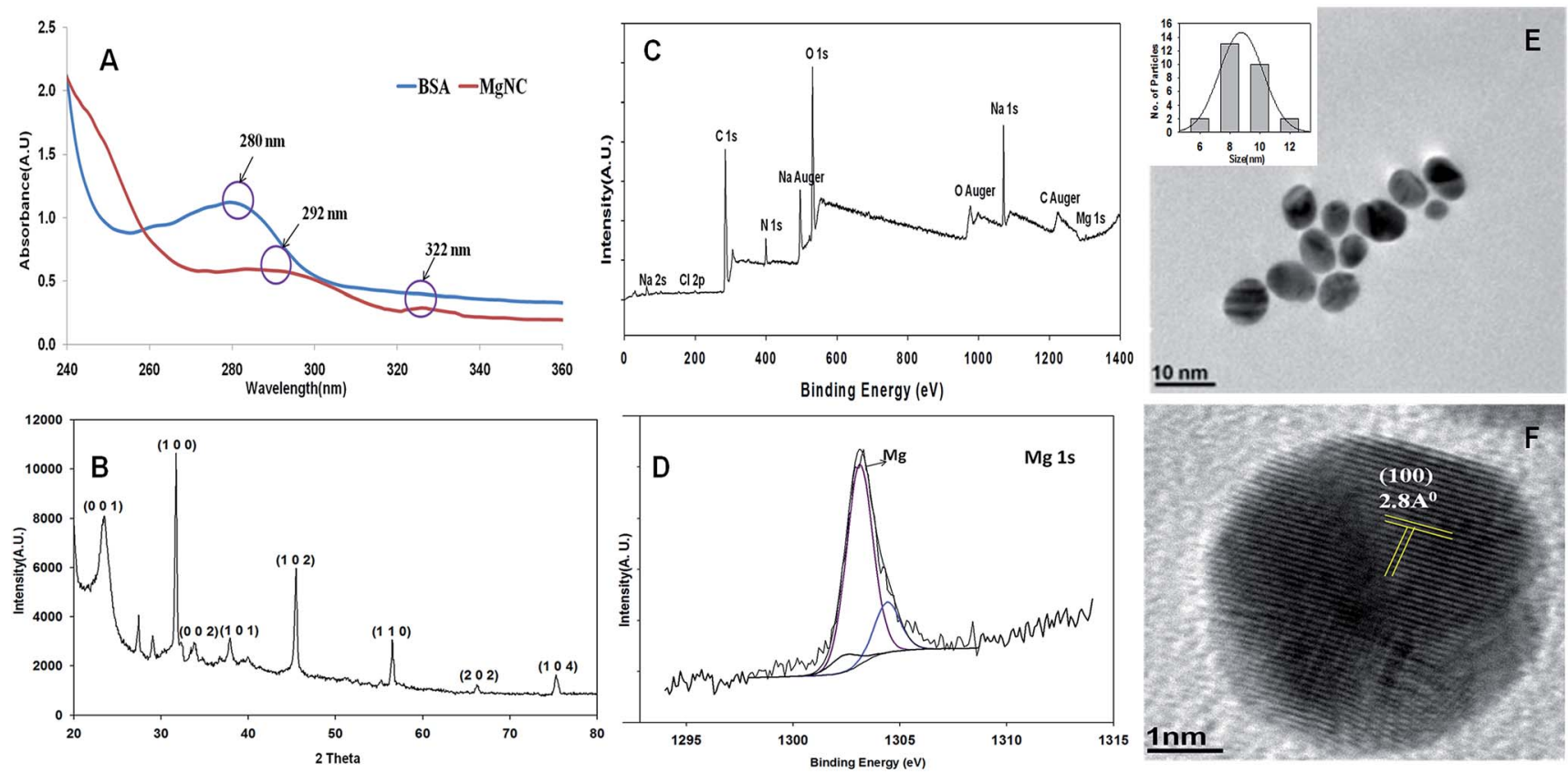

Fig. 2 (A) UV-Vis absorption spectra of BSA (blue line) and MgNC suspension (red line). (B) XRD pattern of MgNC indexed to the hexagonal phase of Mg (JCPDS 04-0770), (C) XPS survey spectrum of MgNC, (D) XPS spectrum in the Mg 1s region of MgNC, (E and F) low and high resolution transmission electron micrograph of $\mathrm{MgNC}$ (inset $\mathrm{E}$ : mean particle size distribution). 
that the product prepared via this route is single phase metallic magnesium.

Elemental identification and oxidation state of magnesium in MgNC were determined by X-ray photoelectron spectroscopy (XPS). The deconvolution of XPS spectra was carried out by CasaXPS software using Gaussian/Lorentzian (GL(30)) line shape and Shirley background correction. The XPS survey spectrum (Fig. 2C) shows the existence of C, N, O, Na, Cl and Mg in the magnesium nanocomplex. Fig. 2D shows the deconvoluted high resolution spectrum of $\mathrm{Mg} 1 \mathrm{~s}$ with one intense peak centered at a binding energy of $1303.1 \mathrm{eV}$ which is assigned to $\mathrm{Mg}(0)$. These results are consistent with XPS measurements performed by Gawande et al. ${ }^{19}$ In addition, two minor peaks are also observed at binding energies of $1304.4 \mathrm{eV}$ and $1302.4 \mathrm{eV}$, which can be associated to the formation of $\mathrm{Mg}-\mathrm{S}$ and $\mathrm{Mg}-\mathrm{COO}$ type species upon reaction with BSA located on the surface of the MgNC cluster. It is further in agreement with our observations of presence of thiol and carboxyl group features in FT-IR. The XPS spectrum (Fig. S2A-C $\dagger$ ) shows the presence of C, $\mathrm{N}$ and $\mathrm{O}$ in the magnesium nanocomplex. The peaks with binding energy of C $1 \mathrm{~s}(284.8 \mathrm{eV}, 288.5 \mathrm{eV}), \mathrm{N} 1 \mathrm{~s}(400 \mathrm{eV}, 402.5 \mathrm{eV})$, and O $1 \mathrm{~s}(533 \mathrm{eV})$ elements are probably due to the presence of BSA protein in $\mathrm{MgNC}$ and taken together, these data validate the successful synthesis of protein-magnesium nanocomplex.

To further investigate the interaction between BSA and magnesium during nanocomplex formation, fluorescence spectra was recorded. As shown in Fig. 3, the luminescence of MgNC revealed distinct excitation and emission maxima at $\lambda_{\mathrm{ex}} \approx 322$ and $\lambda_{\mathrm{em}} \approx 465 \mathrm{~nm}$, respectively. The peak position at $322 \mathrm{~nm}$ of the excitation spectrum was almost alike to the MgNC absorption band in Fig. 3. As the reaction progressed, a gradual increase in the luminescence $\left(\lambda_{\mathrm{em}} \approx 465 \mathrm{~nm}\right)$ from the solution was observed. After around $18 \mathrm{~h}$ of reaction, there was no further enhancement in the luminescence and the reaction was considered to be terminated. Quantum yield (QY) of MgNC was determined by using quinine sulfate $(\mathrm{QY}=0.54)$ in $0.5 \mathrm{M}$ sulfuric acid as a reference. ${ }^{18} \mathrm{QY}$ of $\mathrm{MgNC}$ was found to be 0.17 in ethanol. This luminescence of MgNC enabled their observation by in vitro imaging with minimal interference from cellular autofluorescence. Further, in order to probe the role of $\mathrm{NaOH}$ in the synthesis of MgNC, we recorded the fluorescence of complex ( $\mathrm{Mg}+\mathrm{BSA})$, without $\mathrm{NaOH}$, but as expected we did not find any peak at $\lambda_{\mathrm{em}} \approx 465 \mathrm{~nm}$ (Fig. S3A $\dagger$ ). In addition, we also recorded UV-Vis spectra of complex without $\mathrm{NaOH}$ and we found the absorbance peak at the wavelength of $280 \mathrm{~nm}$ only, which is due to presence of BSA (Fig. S3B $\dagger$ ) and no absorbance signature corresponding to MgNC was found. Hence it is confirmed that fluorescence, post addition of $\mathrm{NaOH}$, at $\lambda_{\mathrm{em}} \approx$ $465 \mathrm{~nm}$ and $\lambda_{\mathrm{ex}} \approx 322 \mathrm{~nm}$ is due to formation of MgNC and not from either BSA or pure salt alone or physical mixture of both.

The chemical stability of MgNC was determined at different salt concentrations such as $100 \mathrm{mM} \mathrm{NaCl}$, and PBS buffer at pH 7.5. After $24 \mathrm{~h}$ incubation, the UV-visible absorbance and emission spectrum of MgNC did not show any significant change suggesting high chemical stability of MgNC at varied salt concentrations (Fig. S4†). FT-IR spectrum of the MgNC comparison with BSA, is shown in Fig. S5. $\dagger$ The peaks in the

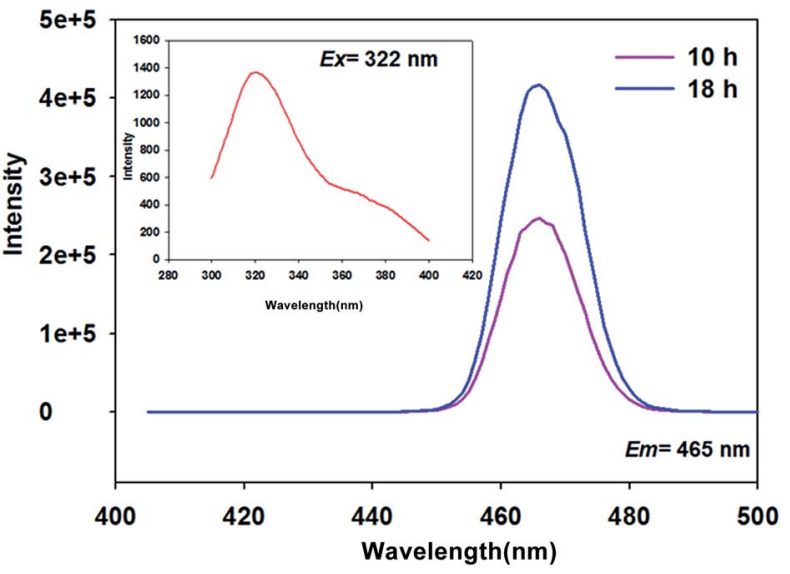

Fig. 3 Fluorescence emission spectra taken at different time intervals (10 $\mathrm{h}$ and $18 \mathrm{~h})$, during the course of reduction.

spectra were assigned as follows: $1635 \mathrm{~cm}^{-1}$ corresponds to a protein with a high proportion of $\alpha$-helix, which is the characteristic amide band of BSA; $1454 \mathrm{~cm}^{-1}$ is attributed to strong primary amine or scissoring while $3429 \mathrm{~cm}^{-1}$ is attributed to primary amines; $2937 \mathrm{~cm}^{-1}$ is ascribed to $\mathrm{C}-\mathrm{H}$ vibration. ${ }^{20 a}$ The disappearance of S-H stretching band $\left(2499 \mathrm{~cm}^{-1}\right)$ of $\mathrm{MgNC}$ is also observed, suggesting the formation of metal-thiol bonds. The MgNC formed in the BSA solution could have been stabilized by a combination of $\mathrm{Mg}-\mathrm{S}$ and $\mathrm{Mg}-\mathrm{COO}^{-}$bonding with the protein (via the amino acid residues in BSA), and the steric protection due to the bulkiness of the protein. ${ }^{16}$ The relationship between the fluorescence intensity and $\mathrm{pH}$ was also studied. The $\mathrm{pH}$ value did not affect the fluorescence intensity of MgNC. We further investigated the photostability of MgNC over a period of 4 months and observed persistent emission (Fig. S6†).

The high luminescence properties and superior photo and chemical stability of these MgNC encouraged us to investigate their possible bioimaging application. The A549 cell line was chosen to assess bio-compatibility using MTT assay at two different concentrations (50 and $100 \mu \mathrm{g} \mathrm{mL}{ }^{-1}$ ) of $\mathrm{MgNC}$. No significant cell death of A549 cells was observed at both the concentrations even after exposure for $24 \mathrm{~h}$ (Fig. S7 $\dagger$ ). Moreover, to substantiate the cytotoxicity, nuclear morphological analysis was also done with MgNC (Fig. S8†). Cells were treated with MgNC at $100 \mu \mathrm{g} \mathrm{mL}{ }^{-1}$ for $24 \mathrm{~h}$ and cell nucleus were stained with DAPI as a control to elicit any apoptotic nuclei. Both MgNC treated and untreated cell's nuclei exhibited normal morphology. No change in nuclear morphology confirmed the non-toxic nature of MgNC even upto $100 \mu \mathrm{g} \mathrm{mL}{ }^{-1}$. This suggested that this nanocomplex is highly biocompatible and does not affect the proper functioning of the nucleus. Based on the aforesaid observations the concentration of $50 \mu \mathrm{g} \mathrm{mL} \mathrm{m}^{-1}$ of MgNC was selected for further experiments.

The A549 and A431 cells $\left(6 \times 10^{4}\right.$ cells per well $)$ were exposed to $50 \mu \mathrm{g} \mathrm{mL}{ }^{-1}$ of $\mathrm{MgNC}$ for $24 \mathrm{~h}$ to investigate their fluorescence imaging capability. Fluorescence microscopy of A549 and A431 cells treated with MgNC shows significant emitting fluorescence from the cells indicating efficient internalization. Interestingly, 
it is evident from the image that the emission arises from the cell nucleus (Fig. 4A and D). Further, nuclear localization was also confirmed by imaging the cells under DIC mode after treated with MgNC (Fig. 4B and E) as other cell components including the plasma membrane were unstained, establishing the potential of the MgNC for nuclear staining. The merged image (Fig. 4C and F) clearly supports that the fluorescent MgNC overlaps with dense nucleus region of DIC image. The A549 and A431 cells retains their normal morphology after MgNC exposure, supporting our observation with MTT. The high specificity of MgNC to cell nucleus may be due presence of free functional groups of denatured BSA over the surface of nanocomplex. ${ }^{1}$

To see the distribution of particles in cells, we performed uptake kinetics study with $50 \mu \mathrm{g} \mathrm{mL}^{-1}$ of MgNC in A549 cells exposed for 1-24 hours. A549 cells were incubated with MgNC for different time ( $1 \mathrm{~h}, 3 \mathrm{~h}, 6 \mathrm{~h}, 12 \mathrm{~h}$ and $24 \mathrm{~h}$ ) and the nuclei are also co-stained with DAPI. The nuclear and cytoplasmic regions are clearly evident under DIC mode of imaging. The merged image (Fig. S9†) clearly supports that fluorescent MgNC and DAPI stained (blue) region overlaps with the dense nuclear region of DIC image. This observation suggest that the internalized MgNC eventually accumulate in cell nucleus. It is also interesting to note that within $1 \mathrm{~h}$ of incubation of MgNC, cells start to internalize the MgNC. It is also obvious from Fig. S9† that some MgNC are dispersed in cytoplasm (at $3 \mathrm{~h}$ and $6 \mathrm{~h}$ ) while most of them have accumulated in nucleus. However, after $12 \mathrm{~h}$ of exposure, MgNC are mostly present in nuclear region and very limited green fluorescence can be seen in cytoplasmic region. This could be due to the fact that after $6 \mathrm{~h}$ cells do not internalize more MgNC, rather accumulate them in nucleus. Similar observation has been reported by Singh et $a l .{ }^{20 b}$ where carboxyfluorescein modified cerium oxide nanoparticles $(<10 \mathrm{~nm})$ widely accumulate in cell nucleus. In order to investigate that the fluorescence is produced by MgNC only and not due to tyrosine and tryptophan residues present in BSA or any other species, we also performed imaging with unreduced MgNC (without $\mathrm{NaOH}$ ). Exposure to cells showed no

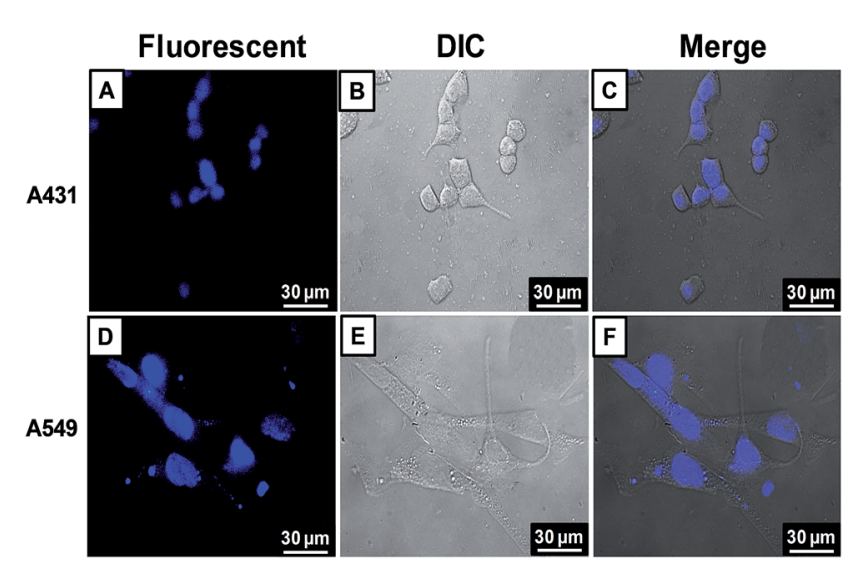

Fig. 4 Fluorescence microscope images of A549 and A431 cell lines after $24 \mathrm{~h}$ exposure with $\mathrm{MgNC}\left(\lambda_{\mathrm{em}}=465 \mathrm{~nm}\right)$; to $\mathrm{A} 431(\mathrm{~A}-\mathrm{C})$ and A549 cells (D-F). The images are pseudo coloured as blue which corresponds to the presence of $\mathrm{MgNC}$ in cell. green fluorescence, as evident by Fig. S10, $\dagger$ this suggests that the fluorescence is due to formation of $\mathrm{MgNC}$ only and not from BSA or other residue.

In addition, to confirm the internalization of MgNC, flow cytometric analysis was carried out. A concentration dependent $\left(10,20,30,40,50 \mu \mathrm{g} \mathrm{mL}{ }^{-1}\right)$ increase in both forward scatter (FSC) as well as in side scatter (SSC) was observed (Fig. S11 $\dagger$ ). Interestingly, the increase in the FSC at concentration $(10,20$, $30 \mu \mathrm{g} \mathrm{mL} \mathrm{m}^{-1}$ ) was substantial and at the same time, SSC augmented at the concentrations of 40 and $50 \mu \mathrm{g} \mathrm{mL}{ }^{-1}$ at $24 \mathrm{~h}$. It clearly suggests that MgNC are internalized via plasma membrane without bilayer disruption, followed by their entrapment in nucleus as increase in forward scatter signifies the internalisation. ${ }^{21 a}$ It can be concluded that the structural organization of surface chemical groups plays a key role in regulating cell-membrane diffusion. Early endosomes are known to gradually mature into late endosomes and eventually transform in lysosomes. ${ }^{21 b}$ It may possibly be due to the "evasion" of MgNC from the lysosomes and ability to move towards the cell nucleus, an advantage compared to other nanoparticles. The aforesaid observations clearly suggest that these ultrasmall particles mainly follow an endosomal pathway for internalization and are ultimately transferred to nucleus.

In conclusion, we have demonstrated a simple method for preparing water-soluble blue green emitting fluorescent MgNC that exhibit superior fluorescence, high chemical and photo stability. The application of MgNC could be as fluorescent nanoprobe to stain nuclei. Further, these non-toxic MgNC could be used a potential drug carrier and DNA transfecting agents. It is expected that more characterisation will unravel the other possible bio-applications of these photo-stable MgNC.

\section{Acknowledgements}

This work was supported by the grant CENTRA from Gujarat Institute Chemical Technology, Ahmedabad, India (Grant No.: ILS/GICT/2013/003) and the Indo-US Science and Technology Forum (IUSSTF/JC-Nanomedicine for Head \& Neck Cancer/342012/2013-14), New Delhi. XPS characterization was performed using EMSL, a DOE Office of Science User Facility sponsored by the Office of Biological and Environmental Research and located at Pacific Northwest National Laboratory. The fellowship of R. Purohit is supported by IIT madras (Gr. No.: CHY1112276DSTXTPRA/R27071).

\section{References}

1 V. Venkatesh, A. Shukla, S. Sivakumar and S. Verma, ACS Appl. Mater. Interfaces, 2014, 6, 2185-2191.

2 D. H. M. Dam, J. H. Lee, P. N. Sisco, D. T. Co, M. Zhang, M. R. Wasielewski and T. W. Odom, ACS Nano, 2012, 4, 3318-3326.

3 (a) L. Shang, S. Dong and G. U. Nienhaus, Nano Today, 2011, 6, 401; (b) L. Shang, R. M. Dorlich, S. Brandholt, R. Schneider, V. Trouillet, M. Bruns, D. Gerthsen and G. U. Nienhaus, Nanoscale, 2011, 3, 2009-2014; (c) P. Bian, J. Zhou, Y. Liu and Z. Ma, Nanoscale, 2013, 5, 6161-6166. 
4 W. C. Chan, D. J. Maxwell, X. Gao, R. E. Bailey, M. Han and S. Nie, Curr. Opin. Biotechnol., 2002, 13, 40-46.

5 R. Hardman, Environ. Health Perspect., 2006, 114, 165-172.

6 (a) J. R. Roberts, J. M. Antonini, D. W. Porter, R. S. Chapman, J. F. Scabilloni and S. H. Young, Part. Fibre Toxicol., 2013, 10, 1-17; (b) A. S. Karakoti, R. Shukla, R. Shanker and S. Singh, Adv. Colloid Interface Sci., 2015, 215, 28-45.

7 J. Zheng, C. A. Nicovich and R. M. Dickson, Annu. Rev. Phys. Chem., 2007, 58, 409.

8 H. Kawasaki, K. Hamaguchi, I. Osaka and R. Arakawa, Adv. Funct. Mater., 2011, 21, 3508.

9 H. W. Duan and S. M. Nie, J. Am. Chem. Soc., 2007, 129, 2412. 10 H. X. Xu and K. S. Suslick, Adv. Mater., 2010, 22, 1078.

11 (a) T. Vosch, Y. Antoku, J. C. Hsiang, C. I. Richards, J. I. Gonzalez and R. M. Dickson, Proc. Natl. Acad. Sci. U. S. A., 2007, 104, 12616; (b) N. Zhao, H. G. Bagaria, M. S. Wong and Y. Zu, J. Nanobiotechnol., 2011, 9, 1-12.

12 (a) Y. Pei, Y. Cao and X. C. Zeng, J. Am. Chem. Soc., 2008, 130, 7830; (b) T. Chen, Y. Hu, Y. Cen, X. Chu and Y. Lu, J. Am. Chem. Soc., 2013, 135(31), 11595-11602.

13 (a) D. M. Chevrier, A. Chatt and P. J. Zhang, Nanophotonics, 2012, 6, 064504; (b) Z. Wang, R. Zhang, Z. Wang, H. F. Wang, Y. Wang, J. Zhao, F. Wang, W. Li, G. Niu, D. O. Kiesewetter and X. Chen, ACS Nano, 2014, 8(12), 12386-12396; (c) J.-H. Choi, H.-J. Hwang, S. W. Shin, J.-W. Choi, S. H. Um and B.-K. Oh, Nanoscale, 2015, 7, 9229-9237; (d) E. Ju, Z. Liu, Y. Du, Y. Tao, J. Ren and
X. Qu, ACS Nano, 2014, 8(6), 6014-6023; (e) S. Singh, V. D'Britto, A. A. Prabhune, C. V. Ramana, A. Dhawan and B. L. V. Prasad, New J. Chem., 2010, 34, 294-301.

14 (a) H. Kawasaki, K. Hamaguchi, I. Osaka and R. Arakawa, Adv. Funct. Mater., 2011, 21, 3508-3515; (b) N. Goswami, A. Giri, M. S. Bootharaju, P. L. Xavier, T. Pradeep and S. Kumar Pal, Anal. Chem., 2011, 83, 9676-9680.

15 C. Sissi and M. Palumbo, Nucleic Acids Res., 2009, 37(3), 702711.

16 G. J. He, W. F. Liu and Y. B. Yan, Int. J. Mol. Sci., 2011, 12, 2901-2916.

17 (a) S. B. Kalidindi and B. R. Jagirdar, Inorg. Chem., 2009, 48(10), 4524-4529; (b) I. Haasa and A. Gedanken, Chem. Commun., 2008, 1795-1797.

18 A. M. Brouwer, Pure Appl. Chem., 2011, 83, 2213-2228.

19 M. B. Gawande, P. S. Branco, K. Parghi, J. J. Shrikhande, R. K. Pandey, C. A. A. Ghumman, N. Bundaleski, O. M. N. D. Teodoro and R. V. Jayaram, Catal. Sci. Technol., 2011, 1, 1653-1664.

20 (a) S. Singh, U. M. Bhatta, P. V. Satyam, A. Dhawan, M. Sastrya and B. L. V. Prasad, J. Mater. Chem., 2008, 18(22), 2601-2606; (b) S. Singh, A. Kumar, A. Karakoti, S. Seal and W. T. Self, Mol. BioSyst., 2010, 6(10), 1813-1820. 21 (a) H. Suzuki, T. Toyooka and Y. Ibuki, Environ. Sci. Technol., 2007, 41, 3018-3024; (b) J. P. Luzio, P. R. Pryor and N. A. Bright, Nat. Rev. Mol. Cell Biol., 2007, 8, 622-632. 